\title{
GÉRALDINE DUTHÉ*
}

\section{Recrudescence du paludisme au Sénégal : la mesure de la mortalité palustre à Mlomp}

\begin{abstract}
La question du paludisme et notamment de sa recrudescence en Afrique est un sujet d'actualité d'une grande importance, tant pour la recherche que pour l'action. Géraldine Duthé nous propose, à partir de données consolidées par la méthode de l'autopsie verbale et des tests parasitologiques, l'étude des tendances (19852003) de la mortalité par paludisme dans une communauté rurale du Sénégal. Ces données précises sur les causes de mortalité constituent une source originale et rare en Afrique qui permet de mesurer la recrudescence de la maladie favorisée par le développement de la chimiorésistance. Cela montre l'intérêt incontestable d'une étude localisée pour une meilleure connaissance des tendances de la mortalité par paludisme en Afrique.
\end{abstract}

La recrudescence du paludisme est actuellement une entrave importante à la transition sanitaire dans les pays du Sud, et plus particulièrement en Afrique au sud du Sahara où cette maladie est l'une des principales causes de décès des enfants de moins de cinq ans. La mortalité palustre est toutefois difficile à étudier dans cette région du monde en raison du manque de données démographiques et sanitaires, surtout en milieu rural. Les sites de suivi démographique permettent en partie de pallier ce déficit mais en l'absence de données médicales, cette cause de décès reste malaisée à diagnostiquer. Depuis 1985, le site de Mlomp au Sénégal fournit des données sur les causes de décès déterminées à partir d'autopsies verbales complétées par des résultats de tests parasitologiques. Situé dans une zone où le paludisme est endémique et où l'offre de soins locale est assez développée, Mlomp est le lieu d'études médicales et épidémiologiques sur la recrudescence du paludisme et l'efficacité des traitements disponibles. Ce site constitue un cadre particulièrement privilégié pour étudier la mortalité palustre. Après un rappel de l'évolution du contexte épidémiologique du paludisme et de ses conséquences sur la mortalité en Afrique, nous estimons la mortalité palustre à Mlomp en tenant compte des

\footnotetext{
* Institut national d'études démographiques, Paris.
} 
diagnostics établis mais aussi des données fournies pour déterminer ces diagnostics. Ainsi, nous tentons de mesurer les conséquences de la recrudescence palustre sur la mortalité générale de cette population suivie.

\section{Le paludisme en Afrique au sud du Sahara}

\section{Le paludisme, un fléau ancien qui sévit toujours dans les pays du Sud}

Le paludisme à Plasmodium falciparum est une maladie parasitaire mortelle dont l'épidémiologie est extrêmement variable selon les régions : elle dépend du climat, de l'environnement et de l'aménagement du territoire. Son impact sur la morbidité et la mortalité dépend de ces caractéristiques qui jouent sur la fréquence et l'intensité de l'exposition. Dans les zones de faible endémie ou à caractère épidémique, le paludisme peut tuer à tous les âges. Dans les zones endémiques, la mortalité palustre est élevée mais touche essentiellement les jeunes enfants ; les individus acquièrent avec l'âge une prémunition qui permet d'éviter le décès.

Très ancienne, cette maladie a été éradiquée en Europe dans les années 1940 comme dans les zones littorales de France métropolitaine par des pulvérisations massives au DDT. Au début des années 1950, le paludisme reste un problème de santé dans les régions intertropicales de la planète, soit pour la plupart des pays du Sud. Mais l'efficacité des insecticides et surtout d'un médicament très bon marché, la chloroquine, laissait espérer l'éradication mondiale de la maladie. La quasi-disparition du paludisme en deux ans au Sri Lanka est à ce titre spectaculaire puisqu'elle aurait permis d'allonger de douze ans l'espérance de vie de la population ${ }^{(1)}$.

Mais des résistances liées à une utilisation massive des produits sont apparues : les moustiques ont développé des résistances aux insecticides - qui se sont par ailleurs révélés toxiques pour les populations - et les parasites aux médicaments antipaludiques. En 1961, des souches de parasites résistantes à la chloroquine émergent presque simultanément en Asie et en Amérique tropicales (Payne, 1987). Du fait de la pauvreté des pays touchés par le paludisme, la chloroquine a continué à constituer le traitement de base et la chloroquinorésistance est apparue sur le continent africain en 1978 à l'Est et une décennie plus tard à l'Ouest (Baudon, 2000 ; Amat-Roze, 2002). Parallèlement, se sont développées selon les régions des résistances des parasites aux autres médicaments antipaludéens utilisés en traitement de deuxième ou troisième intention, comme la quinine ou la sulfadoxine-pyriméthamine ${ }^{(2)}$ (Sibley et al., 2001 ; Gregson et Plowe, 2005).

(1) L'ampleur de l'impact de l'éradication du paludisme sur la baisse de la mortalité au Sri Lanka est néanmoins débattue (Molineaux, 1985 ; Langford, 1996).

(2) Fansidar®. 
Le lancement en 1998 du projet Roll Back Malaria (Faire reculer le paludisme) de l'OMS marque le tournant de la lutte contre le paludisme vers une stratégie prenant en compte les différents facteurs en jeu : clinique, biologique mais aussi socioculturel et économique. La prévention est renforcée - avec notamment l'utilisation de moustiquaires imprégnées d'insecticides - et de nouveaux traitements sont mis en place dans les pays qui utilisent encore la chloroquine bien qu'elle ne soit plus efficace ${ }^{(3)}$. Dans le domaine thérapeutique, l'association de plusieurs molécules permettant de minimiser les risques de résistance paraît la plus prometteuse (Trape, 2001). L'OMS recommande actuellement une multi-thérapie comprenant de l'artémisinine $\left(\mathrm{ACT}^{(4)}\right)$ mais ce type de traitement coûte beaucoup plus cher ${ }^{(5)}$ (OMS, 2005). Il est donc plus difficilement accessible pour les populations des pays en développement (Agnamey et al., 2005 ; Marquet, 2003).

\section{L’impact du paludisme sur la mortalité en Afrique}

En Afrique au sud du Sahara, la population est, au deux tiers, exposée au paludisme. Environ $80 \%$ des décès palustres surviennent dans cette région du monde, touchant principalement les enfants de moins de cinq ans (OMS, 2005). En 2000, le paludisme causerait entre $15 \%$ et $20 \%$ de la mortalité infanto-juvénile, ce qui est considérable, mais l'estimation du nombre de décès dus au paludisme sur ce continent est loin d'être aisée ${ }^{(6)}$ (Rowe et al., 2006 ; Smith et al., 2004, 2006 ; Snow et al., 1999a, 1999b ; MARA/ARMA, 1999).

La mortalité a augmenté dans les dernières décennies en Afrique subsaharienne. Le sida en est bien sûr la première cause mais la recrudescence du paludisme a aussi contribué à cette détérioration sanitaire (OMS, 2005). Malgré certaines limites ${ }^{(7)}$, les enquêtes représentatives au niveau national comme les enquêtes démographiques et de santé (EDS) permettent d'évaluer les tendances générales de la mortalité dans l'enfance (Barbieri, 1989) : elles montrent que la mortalité des enfants a augmenté dans de nombreux pays durant les années 1990, y compris dans des pays d'Afrique moins touchés par l'épidémie de sida comme le Sénégal où le paludisme représente la première cause de morbidité et de mortalité chez les enfants de moins de cinq ans (Ndiaye

(3) L'OMS recommande d'abandonner la chloroquine comme traitement de première ligne lorsque la résistance du Plasmodium falciparum dépasse le seuil de $25 \%$ (proportion d'échecs de soins).

(4) Artemisinin-based combination therapy.

(5) Au début des années 2000, le coût d'un traitement antipaludéen à base de chloroquine est de $0,11 €$ pour un adulte, celui à base de sulfadoxine-pyriméthamine de $0,18 €$, et ceux combinant artesunate avec de l'amodiaquine ou de la sulfadoxine-pyriméthamine coûtent entre 0,87 et $1,3 €$ (Adjuik et al., 2004).

(6) Ces estimations sont basées sur des études locales qui permettent d'estimer selon les régions du continent les populations à risque de transmission et le risque de mourir du paludisme. Selon les études, l'estimation du nombre de décès correspond à la mortalité directement attribuable au paludisme ou à la mortalité induite par cette maladie (cause principale, associée ou indirecte).

(7) En termes de représentativité, fiabilité des déclarations et des informations rétrospectives, biais méthodologiques. 
et Ayad, 2006). Le poids de la mortalité palustre dans la remontée de la mortalité dans l'enfance au cours des années 1990 est cependant difficilement mesurable, faute de statistiques nationales des causes de décès.

En effet, dans la plupart des pays africains, l'état civil se limite souvent aux grandes villes : la majorité des enfants ne sont pas enregistrés dès leur naissance (Unicef, 2002) et l'enregistrement des décès est parcellaire, avec des âges au décès déclarés qui ne sont pas toujours fiables (Lohlé-Tart et François, 1999 ; Hill, 1999). En outre, pour étudier les causes de décès, les données des infrastructures sanitaires sont déficientes : en milieu rural, la majorité des décès a lieu sans qu'un médecin ait vu le malade avant sa mort ou qu'une autopsie ait pu être faite par la suite ${ }^{(8)}$. Au total, seuls quatre pays d'Afrique sub-saharienne fournissaient en 2001 des données nationales de qualité sur les causes de décès ${ }^{(9)}$ (Mathers et al., 2006).

\section{L'apport des sites de suivi démographique pour étudier la mortalité palustre}

Peu de sources de données permettent donc d'étudier de façon précise la mortalité, surtout en milieu rural. Le suivi démographique d'une population est une méthode de collecte qui s'est développée pour disposer de données riches et précises (Pison, 2005 ; Delaunay, 2002). Le suivi s'appuie sur des enquêtes à passages répétés faisant suite au recensement initial d'une population délimitée géographiquement. Il ne permet pas de fournir des données représentatives au niveau national, mais l'épidémiologie du paludisme étant fortement déterminée par des caractéristiques locales, son étude est bien adaptée à ce type de collecte.

Les sites de suivi démographique (SSD) peuvent fournir des informations détaillées sur la mortalité avec un âge précis au décès et la cause probable du décès lorsqu'il fait l'objet d'une autopsie verbale : ce questionnaire consiste à interroger les proches de l'individu décédé pour recueillir des informations sur la maladie, le traitement et les symptômes. Le médecin est ensuite sollicité pour l'interprétation des informations recueillies et la détermination des causes probables du décès. Cette méthode a commencé à être utilisée dans les années 1950 (Biraud, 1956) ; elle s'est considérablement développée et est aujourd'hui appliquée dans de nombreux sites d'Afrique sub-saharienne et d'Asie (Adjuik et al., 2006 ; Indepth, à paraître).

Or, la classification des causes médicales de décès est complexe, même dans un contexte où elles sont systématiquement diagnostiquées et enregistrées (Meslé, 2002). Très informative, cette méthode présente des limites qui font l'objet de beaucoup d'investigations de la part des chercheurs qui travaillent sur ce type de données; en témoigne l'abondante littérature scientifique qui

(8) Au Burkina Faso par exemple, seulement 1 décès sur 12 serait enregistré dans les formations sanitaires (Baya, 2004).

(9) Maurice, les Seychelles, l'Afrique du Sud et le Zimbabwe. 
lui est consacrée depuis la fin des années 1980 (Garenne et Fontaine, 1988 ; Snow et al., 1992 ; Chandramohan et al., 1994 ; Anker, 1997 ; Fauveau, 2002 ; Chandramohan et al., 2005 ; Garenne et Fauveau, 2006 ; Soleman et al., 2006 ; Setel et al., $\left.2006^{(10)}\right)$.

La fiabilité de la méthode dépend de différents facteurs allant de la collecte à la classification de la cause - choix du répondant, questionnaire utilisé, expérience des médecins en charge du diagnostic, etc. - mais elle dépend aussi beaucoup de la cause de décès elle-même. Létude de la performance de la méthode se mesure généralement en termes de sensibilitée ${ }^{(11)}$ et de spécificitée $e^{(12)}$ (Anker, 1997 ; Chandramohan et al., 2001). Ces indices permettent d'évaluer l'écart qui peut exister entre la cause réelle du décès et la cause diagnostiquée par la méthode, cet écart pouvant avoir des conséquences importantes sur les niveaux de mortalité par cause de décès (Maude et Ross, 1997). Généralement, les décès faisant suite à un accident ou à une maladie dont la symptomatologie est très caractéristique comme la rougeole ou la rage sont bien déterminés ; mais il n'existe pas de signe pathognomonique du paludisme (Rogier et al., 2005) : fièvre, anémie, coma, détresse respiratoire ou troubles neurologiques sont des signes que l'on peut retrouver chez une personne atteinte d'une autre pathologie (Marsh et al., 1996 ; Berkley et al., 1999). La méthode de l'autopsie verbale serait donc moins fiable pour diagnostiquer les décès palustres. Les études locales qui ont pu comparer les diagnostics établis par le biais de cette méthode avec des diagnostics cliniques montrent qu'elle est peu sensible et modérément spécifique (Rowe, 2005 ; Anker et al., 1999 ; Todd et al., 1994 ; Snow et al., 1992). Dans la pratique, il est souvent impossible de mesurer précisément ces indices puisque la cause réelle de décès n'est pas connue.

Ainsi, la mesure de la mortalité palustre en Afrique au sud du Sahara est soumise à un double problème : la déficience des données sur les causes de décès de manière générale et la moins bonne validité des données existantes à partir des autopsies verbales. Pourtant, bien qu'elles soient limitées, les estimations actuelles de la mortalité palustre en Afrique se basent essentiellement sur ces données collectées au niveau local (Rowe et al., 2006).

\section{Le site de suivi démographique et sanitaire de Mlomp}

\section{Suivi d'une population rurale du Sénégal depuis 1985}

Au Sud-Ouest du Sénégal, la population de Mlomp fait l'objet d'un suivi démographique et sanitaire depuis 1985 (Pison et al., 2002). Le suivi de ce site

(10) Un bulletin de l'OMS - 84(3) - paru en 2006 sur l'estimation de la mortalité dans les pays en développement est en partie consacré à cette méthode.

(11) La sensibilité de la méthode pour le diagnostic de cause $x$ est la proportion de décès diagnostiqués cause $x$ parmi les décès réellement dus à cette cause $x$.

(12) La spécificité de la méthode pour le diagnostic de cause $x$ est la proportion de décès non diagnostiqués cause $x$ parmi les décès réellement non dus à cette cause $x$. 
a été mis en place pour mesurer les niveaux et les tendances démographiques dans une population rurale d'Afrique sub-saharienne. En 1985, deux observatoires de population, ceux de Niakhar (Garenne et Cantrelle, 1997) et Bandafassi (Pison et al., 1997), assuraient déjà un suivi démographique dans le CentreOuest et au Sud-Est du Sénégal. Mlomp permettait ainsi de mieux couvrir la diversité démographique du pays (carte 1).

Carte 1. Localisation de Mlomp et des deux autres sites ruraux de suivi démographique et sanitaire du Sénégal

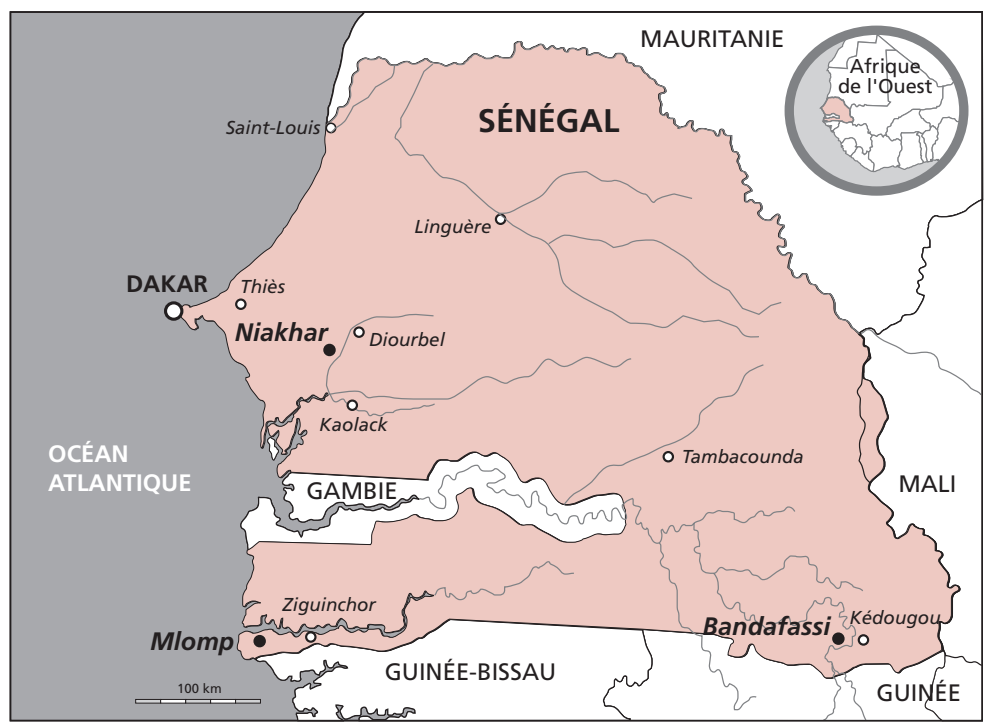

Depuis son recensement initial en 1985, la population de Mlomp est enquêtée annuellement. À chaque passage, les événements démographiques survenus dans l'année sont relevés : naissances, décès, suivi des unions et migrations. La méthode de l'autopsie verbale est utilisée pour aider au diagnostic des causes biomédicales de décès.

\section{Les recherches menées sur le paludisme à Mlomp}

Le climat subtropical de la région de Mlomp est caractérisé par l'alternance d'une saison des pluies (« l'hivernage », de juin à octobre) et d'une saison sèche. Le paludisme à Plasmodium falciparum y est mésoendémique : la transmission est effective toute l'année mais elle est particulièrement intense durant 6 à 8 mois $^{(13)}$. Les enfants sont les seuls à être exposés aux formes graves de la maladie, qui peuvent entraîner le décès. 
Différents programmes de recherche médicale sont menés en partenariat avec le dispensaire du village depuis la fin des années 1980, notamment sur la chloroquino-résistance et l'efficacité des traitements antipaludéens. Beaucoup de patients qui consultent au dispensaire pour un accès de fièvre sont soumis au test de la goutte épaisse permettant de mesurer la densité parasitaire du Plasmodium falciparum dans le sang et de diagnostiquer un accès palustre.

La mise en commun de ces projets a permis de mettre en évidence l'impact de la résistance à la chloroquine sur la mortalité attribuable au paludisme en Afrique de l'Ouest (Trape et al., 1998) puis de confirmer l'efficacité de la multithérapie ACT (Adjuik et al., 2002) et son impact sur la baisse de la mortalité (Cissé et al., 2005). Les deux premières études sont d'ailleurs très régulièrement citées $^{(14)}$.

À Mlomp, les informations médicales disponibles au dispensaire étant utilisées pour aider au diagnostic des décès survenus dans le cadre du suivi démographique, la validité des diagnostics est grandement améliorée. Pourtant, ces informations ne sont ni complètes ni exhaustives. Connaissant les difficultés liées à la détermination des causes de décès - et plus spécifiquement en ce qui concerne le paludisme - dans le cadre des sites de suivi démographique, il apparaît essentiel d'étudier plus précisément la fiabilité du diagnostic pour mesurer l'évolution de la mortalité palustre depuis 1985 et estimer son impact sur la mortalité générale.

\section{Présentation de Mlomp et de sa population}

La zone de Mlomp couvre une agglomération rurale située dans une partie boisée entourée de rizières. Au $1^{\mathrm{er}}$ janvier 2005, la population suivie était de 8008 personnes appartenant à des ménages regroupés en concessions familiales basées sur le système patrilinéaire. Les maisons sont construites en pisé, recouvertes de tôle ou de paille. Les habitants ne sont pas alimentés en électricité et l'eau consommée provient des nombreux puits de la zone (Pison et al., 2002).

Les habitants de Mlomp sont principalement d'ethnie diola (joola), animistes ou catholiques. Ils parlent diola, qui est la langue locale; beaucoup parlent le wolof, la langue véhiculaire du pays, et le français, langue institutionnelle, est appris à l'école. L'infrastructure scolaire est relativement bien développée et en 2000, 55 \% des femmes de 15 à 49 ans ont fréquenté l'école pendant au moins une année contre moins de 20 \% des Sénégalaises vivant en milieu rural en 2005 (Pison et Enel, 2005 ; Ndiaye et Ayad, 2006).

Tandis que l'indicateur synthétique de fécondité dépassait 6 enfants par femme au début des années 2000 en milieu rural sénégalais (Ndiaye et Ayad, 2006), à Mlomp, les femmes ont eu en moyenne 4 enfants sur la période 19852004. Ce niveau de fécondité est lié à un âge moyen à la première naissance

(14) D'après Google Scholar, en septembre 2008, l'article de Trape et al. a été cité 167 fois et celui de Adjuik et al. 145 fois. 
vivante tardif (23 ans). Celle-ci survient généralement avant le mariage au sein d'unions dans lesquelles les partenaires ne cohabitent pas. Après le mariage, les naissances sont espacées notamment par le recours à la contraception (17\% des femmes de 15-49 ans en 2000) et l'allaitement (durée médiane de 19 mois) (Pison et al., 2001).

Sur la période 1985-2004, l'espérance de vie à la naissance atteint 60,5 ans $^{(15),(16)}$. L'estimation de l'espérance de vie au niveau national reste difficile $^{(17)}$ mais la comparaison avec l'espérance de vie observée dans les deux autres sites ruraux du Sénégal sur la même période montre que la population de Mlomp a une mortalité relativement faible pour une zone rurale sénégalaise. La comparaison de la mortalité avant l'âge de cinq ans aboutit à la même conclusion (tableau 1).

Une enquête rétrospective sur les histoires génésiques des femmes recensées à Mlomp en 1985 a permis de montrer une forte diminution du risque de décéder à partir des années 1960. Cette baisse coïncide avec le développement des infrastructures sanitaires dans la zone (Pison et al., 1993).

Tableau 1. Espérance de vie et mortalité avant cinq ans dans les trois sites ruraux du Sénégal, 1985-2004

\begin{tabular}{|l|c|c|c|}
\hline & Bandafassi & Mlomp & Niakhar \\
\hline Espérance de vie à la naissance $\left(e_{0}\right)$ & 48 ans & 61 ans & 54 ans \\
Mortalité infanto-juvénile $\left.{ }_{5} q_{0}\right)$ & $239 \%$ & $101 \%$ & $202 \%$ \\
\hline Source : Pison et al. (2005).
\end{tabular}

\section{Les infrastructures sanitaires et le dispositif de lutte contre le paludisme}

Mlomp n'échappe pas au manque de moyens sanitaires qui prévaut en zone rurale d'Afrique sub-saharienne. Ainsi, le médecin le plus proche se situe à une dizaine de kilomètres de la zone et l'hôpital régional pourvu d'un service de chirurgie est à une distance de $50 \mathrm{~km}$. Néanmoins, un dispensaire a ouvert en 1961 et une maternité en 1968.

(15) Ce type de suivi démographique est limité par une «fenêtre spatio-temporelle » (Delaunay, 2002) : les individus sont observés à une période et sur un espace donnés avec des entrées en observation au moment du recensement initial par naissance ou immigration et des sorties d'observation par décès ou émigration. Les modèles de durée permettent de retracer la courbe de survie de la population en tenant compte de tous les séjours des individus ayant fait partie de la population au moins à un moment donné sur la période considérée.

(16) La mortalité aux grands âges étant sujette à caution, on ne tient pas compte de la mortalité observée à partir de 80 ans et on applique une espérance de vie à 80 ans théorique ( $e_{80}=4,7$ ans) qui est le standard des Nations unies pour une espérance de vie à la naissance de 60 ans (Nations unies, 2002).

(17) Suite aux estimations de la mortalité avant l'âge de cinq ans fournies par la dernière EDS, la révision 2006 des Nations unies propose pour le Sénégal une espérance de vie de 61,6 ans pour la période 2000-2005, soit 6 ans de plus que dans la révision précédente pour la même période (Nations unies, 2005 et 2007). 
Ces deux infrastructures privées fonctionnent bien et depuis longtemps: sous la responsabilité du médecin-chef du district, une infirmière travaille quotidiennement au dispensaire ${ }^{(18)}$ et deux matrones se relaient pour assurer une permanence à la maternité. En cas de complication, les malades sont évacués par l'infirmière à l'hôpital du district ou par l'ambulance de cet hôpital à l'hôpital régional. Toutes les femmes de Mlomp accouchent à la maternité(19) (Enel et al., 1993). Ainsi, le niveau de mortalité maternelle est plus faible à Mlomp que dans les deux autres sites ruraux du Sénégal ${ }^{(20)}$ (Pison et al., 2000) et la mortalité néonatale est relativement basse : le risque de décéder dans le premier mois de vie est de $27 \%$ en 1985-2004 contre $46 \%$ en milieu rural sénégalais sur la période 1996-2005 (Ndiaye et Ayad, 2006).

Depuis de nombreuses années, des séances mensuelles de pesée $e^{(21)}$ et de vaccinations ${ }^{(22)}$ sont organisées : les enfants de Mlomp sont quasiment tous vaccinés contre les maladies recommandées par les autorités sanitaires, ce qui est exceptionnel ${ }^{(23)}$. Ces séances sont aussi l'occasion de « causeries» où les matrones conseillent les mères sur la santé de leurs enfants. Elles y abordent surtout les thèmes de l'allaitement, de l'hygiène, de la vaccination et la prévention contre les principales maladies non évitables par la vaccination : les infections intestinales et le paludisme.

Pour lutter contre le paludisme, la chloroquine a été utilisée comme traitement de base dès l'ouverture du dispensaire en 1961. En 1975, un important programme de chloroquinisation a été lancé, basé sur un traitement prophylactique des habitants pendant la saison des pluies et un traitement présomptif donné en automédication le plus rapidement possible pour tout cas de fièvre observé chez un enfant. Mais avec l'apparition de la chloroquino-résistance en 1990 et son développement extrêmement rapide, la chimioprophylaxie a été limitée en 1993 aux enfants de moins de 4 ans et aux femmes enceintes (Trape et al., 1998 ; Pison et al., 1993). La chloroquine est restée le traitement présomptif pour les jeunes enfants et la quinine est utilisée en cas de forte fièvre ainsi que

(18) Au début des années 2000, l'infirmière est assistée par un aide infirmier et par une aide laborantine. Le dispensaire est équipé d'une salle de consultations et de soins, d'un laboratoire, d'une pharmacie et de lits d'hospitalisation.

(19) Entre 1985 et 2005, seulement 11 accouchements (soit 0,4\% des accouchements hors fausse couche) qui ont eu lieu au village de Mlomp se sont produits hors de la maternité, dont 5 sur le chemin entre le domicile et la maternité. Dans le pays, seulement 44,6\% des naissances en milieu rural en 2000-2005 ont eu lieu dans un établissement sanitaire (Ndiaye et Ayad, 2006).

(20) En 1985-1998, la mortalité maternelle était de 436 pour 100000 naissances vivantes à Mlomp contre 516 à Niakhar et 826 à Bandafassi (Pison et al., 2000).

(21) Depuis 1969, date de la mise en place au Sénégal du Programme de protection nutritionnelle et sanitaire (PPNS).

(22) Ponctuelles à partir de la fin des années 1960, régulières depuis 1975, elles sont devenues mensuelles en 1982, date de la mise en place au Sénégal du Programme élargi de vaccination (PEV).

(23) Sur l'ensemble des enfants nés à Mlomp entre 1995 et 1999, toujours vivants à 18 mois et restés au village, 98 \% ont été complètement vaccinés avant l'âge de 18 mois, $92 \%$ avant l'âge de 1 an (Duthé, 2006). En 2005, 59 \% des Sénégalais âgés de 12 à 23 ans (48\% des enfants de moins d'un an) répondent à ces critères (Ndiaye et Ayad, 2006). 
pour les adultes. Cependant, la résistance du Plasmodium falciparum à la chloroquine n’a jamais cessé de se développer et dépassait 70 \% en 1997 (Sokhna et al., 1997 ; Trape et al., 2002).

En 1999, une étude internationale financée en partie par l'OMS a été mise en place pour tester une ACT combinant amodiaquine et artesunate dans quatre sites africains (Adjuik et al., 2002) : plusieurs centaines d'enfants âgés de 6 mois à 10 ans ont été soignés au dispensaire de Mlomp, soit par de l'amodiaquine seulement ${ }^{(24)}$, soit par une combinaison d'amodiaquine et d'artesunate, les médicaments ayant été fournis par des laboratoires pharmaceutiques. Les résultats encourageants ont amené les chercheurs à traiter en 2000 toute la population par cette ACT en saison des pluies, et tout au long de l'année depuis 2002. Tous ces changements se font en accord avec les autorités sanitaires et le personnel soignant du dispensaire y participe activement, son adhésion étant de toute façon indispensable au succès de ce programme. Ce nouveau traitement de première intention revient actuellement entre $0,74 €$ pour un enfant de moins de cinq ans et $1,3 €$ pour un adulte (Agnamey et al., 2005), coût auquel il faut ajouter le prix de la consultation $(0,15 €$ pour un adulte, la moitié pour un enfant en 2002) et celui du test sanguin pour détecter le Plasmodium falciparum ( $0,3 €$ en 2002). Bien que ce soit cher pour les villageois, son efficacité permet d'éviter les injections de quinine par voie intramusculaire effectuées en soins de deuxième intention, qui sont plus chères encore.

Parallèlement, l'organisation sanitaire locale incite la population à se munir de moustiquaires imprégnées d'insecticides, surtout lors de la saison des pluies, pour protéger les femmes enceintes, les nouveau-nés et les jeunes enfants.

Les institutions sanitaires sont restées démunies durant toute la décennie 1990, laissant craindre une remontée de la mortalité palustre. Larrivée des nouveaux traitements qui se mettent en place progressivement depuis le début des années 2000 a ensuite permis d'espérer un recul de la mortalité palustre.

\section{Mesure de la mortalité palustre et de son impact sur la mortalité générale depuis 1985}

\section{Protocole de détermination des causes de décès}

À Mlomp, les recueils d'autopsies verbales se font au moment de la collecte annuelle, dans un délai allant de la fin de la période de deuil à une année si le décès est survenu juste après la précédente collecte. Les autopsies verbales sont conduites par des enquêteurs ${ }^{(25)}$ originaires de Mlomp, ce qui permet une

(24) Une première étude menée à la fin des années 1990 avait montré la plus grande efficacité de l'amodiaquine par rapport à la chloroquine dans le traitement du paludisme à Mlomp (Brasseur et al., 1999).

(25) La plupart des autopsies verbales sont conduites par un homme mais les questions liées à la reproduction étant taboues chez les Diola, c'est une femme qui enquête sur les décès des nouveaunés et ceux des femmes d'âge fécond. 
meilleure compréhension linguistique et socioculturelle, et une relation de confiance indispensable au bon déroulement de l'entretien ${ }^{(26)}$ (Chandramohan et al., 2005). Ce sont les enquêteurs qui veillent à trouver le proche ou le parent le plus apte à répondre aux questions sur l'histoire de la maladie et des symptômes. Depuis 1985, le même questionnaire ${ }^{(27)}$ d'autopsie verbale est utilisé pour tous les décès mais il comporte un volet spécial pour les enfants et les femmes en âge de procréer ${ }^{(28)}$. Après l'identification de la personne décédée et du répondant, ce dernier déclare la cause qu'il suppose être à l'origine du décès, les éventuels traitements que le malade a reçus et raconte la maladie. Le questionnaire passe ensuite en revue toute une série de symptômes ${ }^{(29)}$ à détailler s'ils sont signalés par le répondant.

L'apport des informations médicales recueillies auprès des institutions sanitaires locales avec l'accord du médecin-chef du département contribue grandement à augmenter la validité de la méthode. Le protocole de détermination des causes de décès a sensiblement été le même jusqu'en 2003 : un seul et même médecin spécialiste du paludisme déterminait les causes de décès en se rendant à Mlomp à la fin du passage annuel pour consulter les différents registres ${ }^{(30)}$ tenus par l'infirmière du dispensaire et les confronter aux autopsies verbales. Les causes de décès sont codifiées selon la neuvième révision de la Classification internationale des maladies (OMS, 1977). Seule la cause principale du décès est identifiée et saisie.

Pour favoriser les études comparatives, les trois sites ruraux de suivi démographique du Sénégal ont le même protocole depuis 2004. À Mlomp, cela s'est traduit par un changement d'enquêteur, le nouvel enquêteur ayant une formation médicale, et la lecture des autopsies par plusieurs médecins de spécialités différentes, permettant une confrontation des différents diagnostics. Les éventuelles informations médicales disponibles au dispensaire sont désormais reportées sur les autopsies verbales au moment de la collecte pour les médecins qui ont la charge des diagnostics à Dakar. Pour l'étude de la mortalité palustre, nous considérons la période 1985-2003 qui est homogène du point de vue des sources d'information, de sa collecte et de son archivage.

(26) Avant de commencer le questionnaire, l'enquêteur explique les objectifs de l'enquête et demande l'accord oral du répondant.

(27) Le questionnaire est celui qui a été utilisé sur le site de Niakhar en 1985 et il n’a été que légèrement modifié depuis.

(28) Ce volet comporte des questions sur les grossesses précédentes, la dernière grossesse, l'accouchement et l'état de santé de l'enfant.

(29) Fièvre/corps chaud, diarrhée/dysenterie, signes de déshydratation, vomissements, crises convulsives et autres signes neurologiques, difficultés de respirer, toux, boutons, plaies, brûlures, abcès, saignements, œdèmes, ventre gonflé, problèmes urinaires, couleur anormale des urines et des selles, problème aux yeux, douleurs (si décès survenu après l'âge de 2 ans), signes généraux, maladie chronique.

(30) Registre des consultations au dispensaire, registre des décès constatés par l'infirmière avec cause supposée, registre des consultations prénatales et registre des accouchements à la maternité. 


\section{Fiabilité du diagnostic palustre}

Notre analyse se restreint aux décès survenus avant l'âge de 20 ans, considérant qu'au-delà, la probabilité d'observer un décès palustre est très faible du fait de la prémunition acquise. De 1985 à 2003, aucun décès palustre n’a d'ailleurs été diagnostiqué au-delà de 20 ans et plus de $80 \%$ des décès diagnostiqués palustres sont survenus avant l'âge de cinq ans (tableau 2).

Tableau 2. Répartition des décès diagnostiqués palustres par groupe d'âges à Mlomp, 1985-2003

\begin{tabular}{|l|c|c|c|}
\hline \multicolumn{1}{|c|}{ Groupe d'âges } & Nombre de décès & $\%$ & \% cumulé \\
\hline 0 an & 8 & 13 & 13 \\
$1-4$ ans & 41 & 69 & 82 \\
$5-9$ ans & 8 & 13 & 95 \\
$10-19$ ans & 3 & 5 & 100 \\
\hline Ensemble & 60 & 100 & \\
\hline \multicolumn{2}{|l}{ Source : base de données Mlomp, 2005. } \\
\hline
\end{tabular}

Si on considère les causes de décès telles qu'elles sont diagnostiquées, le risque de décéder du paludisme avant l'âge de 20 ans a beaucoup varié au fil des années, et son évolution reflète le calendrier des changements épidémiologiques et thérapeutiques décrits précédemment : la mortalité est quasi nulle jusqu'au début des années 1990, et bien qu'elle soit assez variable à partir de 1992, elle se maintient à un niveau moyen de plus de $30 \%$ au milieu de la décennie et diminue ensuite progressivement (figure 1).

Figure 1. Évolution annuelle du risque de décéder du paludisme avant l'âge de 20 ans, Mlomp, 1985-2003

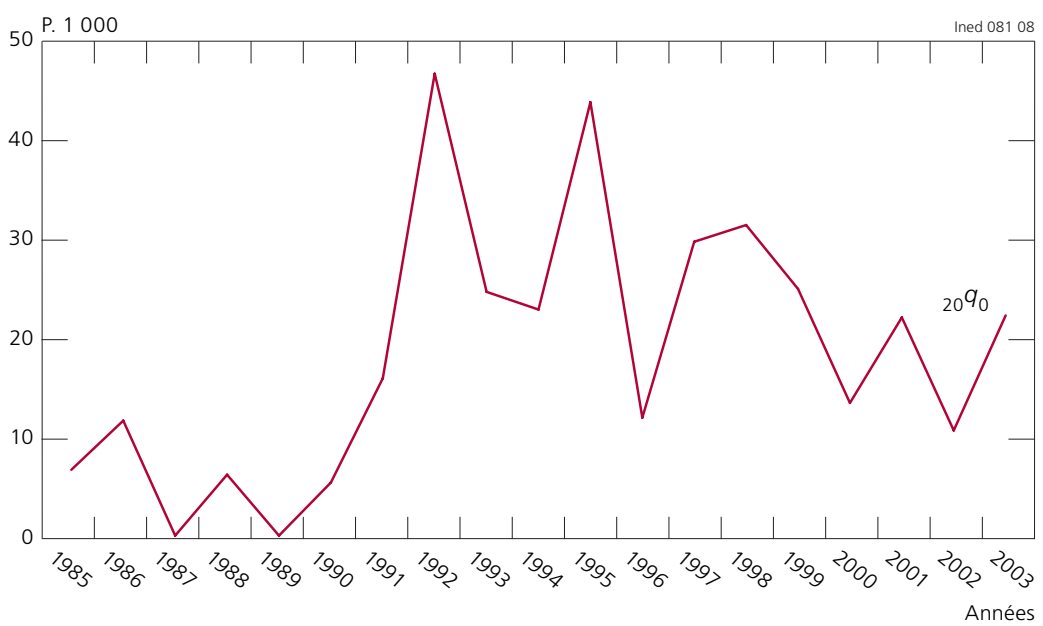

Source : base de données Mlomp, 2005. 
On sait que la connaissance et l'expérience du médecin en charge de déterminer les causes de décès peuvent influencer ce dernier au moment de la décision de classer ou non un décès comme palustre (Desgrées du Loû et al., 1996). L'hypothèse d'une bonne fiabilité du diagnostic palustre à Mlomp liée à l'apport des informations médicales permet de valider ces données. D’un point de vue étiologique, si la présence du parasite dans le sang atteste bien d'un accès palustre, cela ne signifie pas pour autant que le paludisme soit la cause principale du décès. Par ailleurs, les tests de goutte épaisse n'ont été effectués qu'à partir de 1989 et ne sont pas disponibles pour tous les enfants décédés. On peut donc se demander si des décès palustres n'ont pas été diagnostiqués. En outre, les décès ne sont pas tous clairement diagnostiqués et ceux de cause mal définie ne sont pas indépendamment distribués selon les causes de décès, plus ou moins faciles à diagnostiquer (Desgrées du Loû et al., 1996 ; Kahn et al., 2000). Sur la période 1985-2003, un tiers de l'ensemble des décès recensés n'ont pas une cause clairement définie. La proportion est moindre lorsque le décès est survenu avant 60 ans, mais elle représente encore un quart des décès, ce qui est loin d'être négligeable. Avant 15 ans, la moitié des décès de cause mal définie sont caractérisés par des symptômes généraux (fièvre, convulsions, frissons, coma...) (tableau 3).

Tableau 3. Répartition des décès de cause mal définie par grand groupe d'âges, Mlomp, 1985-2003

\begin{tabular}{|l|c|c|c|c|}
\hline & $\begin{array}{c}\text { Moins de } \\
15 \text { ans }\end{array}$ & 15 à 59 ans & $\begin{array}{c}60 \text { ans et } \\
\text { plus }\end{array}$ & Ensemble \\
\hline Part des décès mal définis (en \%) & 25 & 24 & 39 & 32 \\
Dont symptômes généraux & 12 & 4 & 8 & 8 \\
Sénilité, vieillesse & 0 & 0 & 9 & 4 \\
Autres symptômes & 4 & 9 & 9 & 8 \\
Mort subite ou cause inconnue & 9 & 11 & 14 & 12 \\
\hline Nombre de décès mal définis & 105 & 83 & 286 & 474 \\
Nombre total de décès & 426 & 344 & 727 & 1497 \\
\hline Source : base de données Mlomp, 2005.
\end{tabular}

\section{Définition des décès potentiellement liés au paludisme}

Pour estimer les décès potentiellement liés au paludisme, nous distinguons quatre groupes de décès : ceux qui sont diagnostiqués palustres, ceux qui sont diagnostiqués pour une cause autre que palustre, les décès de cause mal définie mais caractérisés par des symptômes et, enfin, les décès de cause inconnue. $\mathrm{Au}$ sein de chaque groupe, nous caractérisons les décès selon la possibilité qu'ils soient dus au paludisme en fonction des informations disponibles (symptomatologie, informations médicales, âge et saison du décès, commentaire du médecin procédant à la détermination de la cause) (tableau 4). 
Tableau 4. Décès potentiellement palustres survenus avant l'âge de 20 ans selon le type de diagnostic, Mlomp, 1985-2003

\begin{tabular}{|c|c|c|c|}
\hline & Possibilité & $\begin{array}{l}\text { Nombre de } \\
\text { décès }\end{array}$ & $\%$ \\
\hline \multicolumn{4}{|l|}{ Décès diagnostiqués palustres } \\
\hline Accès palustre et résultat de goutte épaisse positive & Certain & 27 & 45,0 \\
\hline Accès palustre typique (symptômes, saison, âge) & Présomptif & 26 & 43,3 \\
\hline \multirow[t]{2}{*}{ Accès palustre ou autre cause ou décès mal défini } & Possible & 7 & 11,7 \\
\hline & Total & 60 & 100,0 \\
\hline \multicolumn{4}{|l|}{ Décès diagnostiqués non palustres } \\
\hline En cause associée ou doute & Possible & 26 & 9,3 \\
\hline \multirow[t]{2}{*}{ Pas de doute ou goutte épaisse négative } & Exclu & 253 & 90,7 \\
\hline & Total & 279 & 100,0 \\
\hline \multicolumn{4}{|l|}{ Décès de cause mal définie caractérisés par des symptômes } \\
\hline Fièvres indéterminées ou autres symptômes généraux & Possible & 45 & 62,5 \\
\hline \multirow{2}{*}{$\begin{array}{l}\text { Autre symptôme, pas de doute ou goutte épaisse } \\
\text { négative }\end{array}$} & Exclu & 27 & 37,5 \\
\hline & Total & 72 & 100,0 \\
\hline Décès de cause inconnue & Pas d'indication & 39 & 100,0 \\
\hline
\end{tabular}

Les 60 décès diagnostiqués palustres de 1985 à 2003 sont distingués selon qu'ils sont certains (confirmés par une goutte épaisse positive), présomptifs (accès palustres typiques à un âge et à une saison de l'année propices) ou possibles (cause associée ou concurrente, hésitation à classer dans une catégorie mal définie). Selon cette classification, près de la moitié des décès diagnostiqués palustres sont certains, $43 \%$ sont présomptifs et $12 \%$ sont possibles.

Les décès diagnostiqués non palustres sont classés selon qu'ils pourraient éventuellement être des décès palustres (diagnostic douteux ou paludisme associé) ou au contraire que le diagnostic palustre est exclu (certitude de la cause diagnostiquée ou goutte épaisse négative). Sur 279 décès, plus de $9 \%$ pourraient être dus au paludisme, soit près d'une trentaine de décès. Ces derniers sont surtout des diagnostics de pneumonies ou broncho-pneumonies, d'infections périnatales ou intestinales mal définies, ou encore de méningites. Certaines infections ont en effet des manifestations cliniques proches de l'accès palustre.

Plus de la moitié des décès de cause mal définie mais caractérisés par des symptômes pourraient être liés au paludisme. Sur ces 45 décès, 41 correspondent à des descriptions de symptômes généraux : 30 cas de fièvre, 7 de coma et 4 de convulsions.

Enfin, parmi les décès de cause mal définie, nous distinguons les décès pour lesquels aucune précision n'est disponible. Les décès classés dans cette catégorie résultent généralement d'une absence totale d'informations fournies 
par les autopsies verbales ou le dispensaire. Ces décès ne peuvent pas être liés d'une manière ou d'une autre à telle ou telle cause et leur répartition proportionnelle au sein des autres catégories - certain, présomptif, possible et exclu - apparaît comme étant la méthode de redistribution la plus neutre. Sur la période 19852003, 39 décès survenus avant l'âge de 20 ans sont de cause inconnue.

Le regroupement de tous ces décès nous amène à considérer que la part du paludisme dans la mortalité avant 20 ans sur la période 1985-2003 se situe entre moins de $7 \%$ et près de $32 \%$, soit une fourchette très large entre les décès palustres diagnostiqués de manière certaine et ceux qui pourraient être associés (tableau 5). Par la suite, nous qualifierons ces derniers (soit les décès diagnostiqués de façon certaine, présomptive ou possible) de décès potentiellement palustres.

Tableau 5. Synthèse des décès potentiellement palustres, Mlomp, 1985-2003

\begin{tabular}{|c|c|c|c|c|}
\hline Potentialité & $\begin{array}{l}\text { Nombre de } \\
\text { décès }\end{array}$ & $\begin{array}{l}\text { Nombre après redistribution } \\
\text { des décès de cause inconnue }\end{array}$ & $\%$ & $\%$ cumulé \\
\hline Certain & 27 & 29,6 & 6,6 & 6,6 \\
\hline Présomptif & 26 & 28,4 & 6,3 & 12,9 \\
\hline Possible & 78 & 85,4 & 19,0 & 31,9 \\
\hline Exclu & 280 & 306,6 & 68,1 & 100,0 \\
\hline Cause inconnue & 39 & - & - & \\
\hline Total & 450 & 450,0 & 100,0 & \\
\hline
\end{tabular}

\section{Impact du paludisme sur la mortalité}

Pour étudier l'impact du paludisme sur la mortalité, nous utilisons les taux de mortalité selon les différentes catégories prédéfinies, qui ont l'avantage d'être additifs. Ces taux sont exprimés en personnes-années ${ }^{(31)}$. La figure 2 présente l'évolution par période des taux comparatifs ${ }^{(32)}$ de mortalité avant 20 ans en distinguant les décès potentiellement palustres, les décès de cause inconnue étant redistribués proportionnellement selon l'âge et la période. Quel que soit le degré de potentialité de la mortalité palustre, la distinction entre la première période et la seconde est très nette avec un taux de mortalité potentiellement lié au paludisme qui atteint 3 décès pour mille personnes de moins de 20 ans au cours d'une année. Globalement, on peut donc confirmer qu'il y a eu une augmentation de la mortalité palustre au début des années 1990 qui coïncide bien avec une remontée du niveau de mortalité. Néanmoins, en 1995-1999, le

(31) Le taux de mortalité est le nombre de décès survenus au sein de la population rapporté à la somme des années vécues par les individus durant le suivi.

(32) L'utilisation des taux comparatifs permet d'éliminer l'effet de la structure par âge et de comparer les niveaux observés sur les quatre périodes. Notons que la structure par âge de la population n'a que très peu d'influence sur la variation des taux de mortalité. 
niveau de mortalité a de nouveau baissé tandis que la mortalité palustre n’a pas particulièrement évolué. La mortalité palustre certaine ou présomptive diminue dans la dernière période, mais la possibilité qu'il existe des décès palustres reste importante.

Figure 2. Évolution du taux comparatif de mortalité potentiellement palustre entre 0 et 20 ans par période, Mlomp, 1985-2003 (pour mille personnes-années)

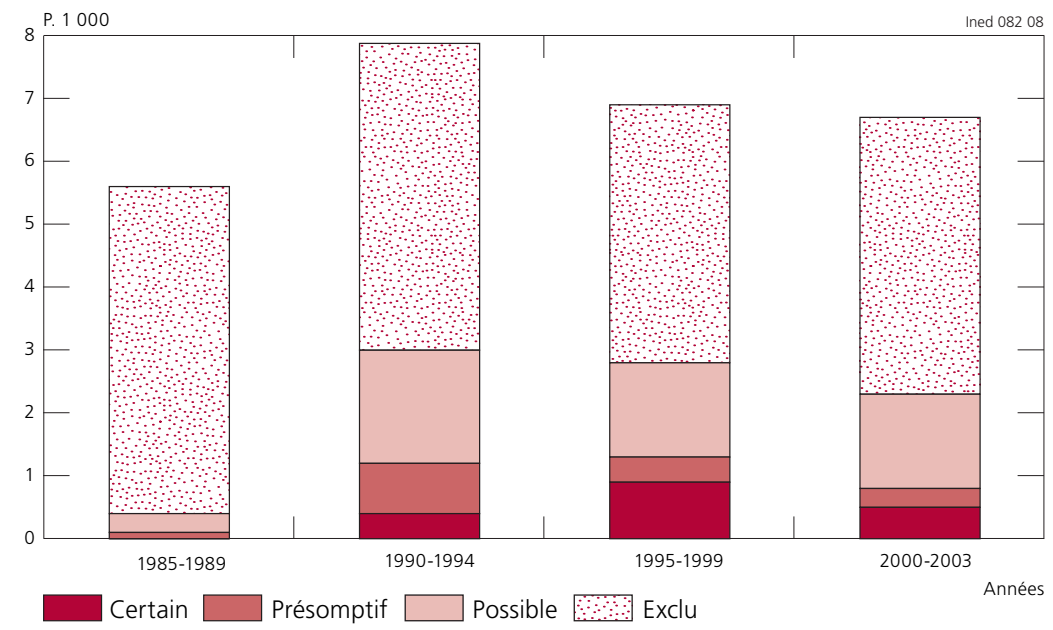

Source : base de données Mlomp, 2005.

Pour estimer l'impact de la mortalité potentiellement palustre, on peut calculer la contribution de chaque catégorie aux variations de l'espérance de vie d'une période à une autre selon le groupe d'âges et le sexe ${ }^{(33)}$. L'espérance de vie à la naissance a en effet beaucoup varié au cours de la période avec une dégradation importante au début des années 1990, surtout pour les femmes dont l'espérance de vie a perdu 7 années entre 1985-1989 et 1990-1994, ce qui est considérable (tableau 6). La mortalité des femmes a augmenté à tous les âges entre ces deux périodes mais c'est la hausse de la mortalité infanto-juvénile qui en est essentiellement responsable. Parce que le protocole d'enquête n'a pas changé au cours du temps et que cette variation ne touche que les filles, il est difficile de conclure à un artefact, mais le fait que la mortalité des femmes soit particulièrement faible sur la première période a probablement accentué l'effet d'une détérioration sanitaire (Duthé, 2006). On peut aussi se demander si le paludisme n'a pas eu un impact plus important chez les jeunes filles que chez les garçons.

(33) En utilisant pour cela l'algorithme proposé par Andreev et al. (2002) qui permet de décomposer la variation d'espérance de vie entre deux périodes selon les contributions propres à l'évolution par âge des différentes causes de décès. 
Tableau 6. Évolution et variations de l'espérance de vie à la naissance par période selon le sexe, Mlomp, 1985-2003 (en années)

\begin{tabular}{|l|c|c|c|c|c|c|c|}
\hline & $1985-1989$ & $1990-1994$ & $1995-1999$ & $2000-2003$ & \multicolumn{3}{|c|}{ Variations } \\
\cline { 2 - 8 } & $(1)$ & $(2)$ & $(3)$ & $(4)$ & $(2)-(1)$ & $(3)-(2)$ & (4)-(3) \\
\hline Hommes & 58,4 & 56,5 & 55,9 & 55,1 & $-1,9$ & $-0,6$ & $-0,8$ \\
Femmes & 68,5 & 61,5 & 64,5 & 63,8 & $-7,0$ & $+3,0$ & $-0,7$ \\
\hline Ensemble & 63,1 & 58,9 & 59,8 & 59,2 & $-4,2$ & $+0,9$ & $-0,6$ \\
\hline Source : base de données Mlomp, 2005.
\end{tabular}

Par souci de clarté, nous avons regroupé les deux premières catégories de décès potentiellement palustres (certains et présomptifs). Pour les garçons, entre 1985-1989 et 1990-1994, la mortalité potentiellement palustre a contribué à diminuer l'espérance de vie dans tous les groupes d'âges, mais cette perte est en grande partie compensée par des progrès sur les autres causes, paludisme exclu. Entre le début et la fin des années 1990, le paludisme continue de faire perdre des années d'espérance de vie mais plus particulièrement dans la période juvénile. En 2000-2003, des progrès sont réalisés sur le paludisme certain et présomptif mais la mortalité potentiellement palustre avant l'âge de 1 an contribuerait encore à diminuer l'espérance de vie (figure 3). Pour les filles, les écarts d'espérance de vie d'une période à l'autre sont bien plus importants. Le paludisme serait responsable d'une baisse de plus de trois années de l'espérance de vie en 1990-1994 par rapport à la première période mais la détérioration n'est pas exclusivement liée à cette recrudescence. À la fin des années 1990, cette perte liée aux autres causes que le paludisme est en grande partie récupérée mais pas celle liée au paludisme certain ou présomptif. Les gains sur le paludisme ne sont récupérés que partiellement en 2000-2003 (figure 4).

Globalement, entre 0 et 20 ans, l'espérance de vie à la naissance des garçons et des filles a perdu plus d'une année entre 1985-1989 et 1990-1994 en raison du paludisme certain ou présomptif. La perte est respectivement de 2,4 et 3,3 ans si on considère aussi le paludisme possible. À la fin des années 1990, les garçons ont encore perdu 6 mois tandis que les filles ont récupéré plus de 8 mois. Au début des années 2000, la contribution potentielle du paludisme n’a que très peu changé pour les garçons et les filles ont gagné une année (tableau 7).

Tableau 7. Contribution du paludisme aux variations de l'espérance de vie à la naissance entre les périodes, selon le sexe et de 0 à 20 ans à Mlomp

\begin{tabular}{|l|c|c|c|c|c|c|}
\hline \multirow{2}{*}{} & \multicolumn{6}{|c|}{ Écart (en années) } \\
\cline { 2 - 7 } & \multicolumn{3}{|c|}{ Garçons } & \multicolumn{3}{c|}{ Filles } \\
\cline { 2 - 8 } & $(2)-(1)$ & (3)-(2) & (4)-(3) & (2)-(1) & (3)-(2) & (4)-(3) \\
\hline Paludisme certain ou présomptif & $-1,1$ & $-0,4$ & $+0,6$ & $-1,2$ & $-0,1$ & $+0,9$ \\
Paludisme possible & $-1,3$ & $-0,1$ & $-0,4$ & $-2,1$ & $+0,8$ & $+0,1$ \\
Paludisme exclu & $+1,9$ & $+0,4$ & $-0,3$ & $-2,3$ & $+2,1$ & $-0,6$ \\
\hline
\end{tabular}


Figure 3. Contribution du paludisme par groupe d'âges aux variations de l'espérance de vie masculine à Mlomp

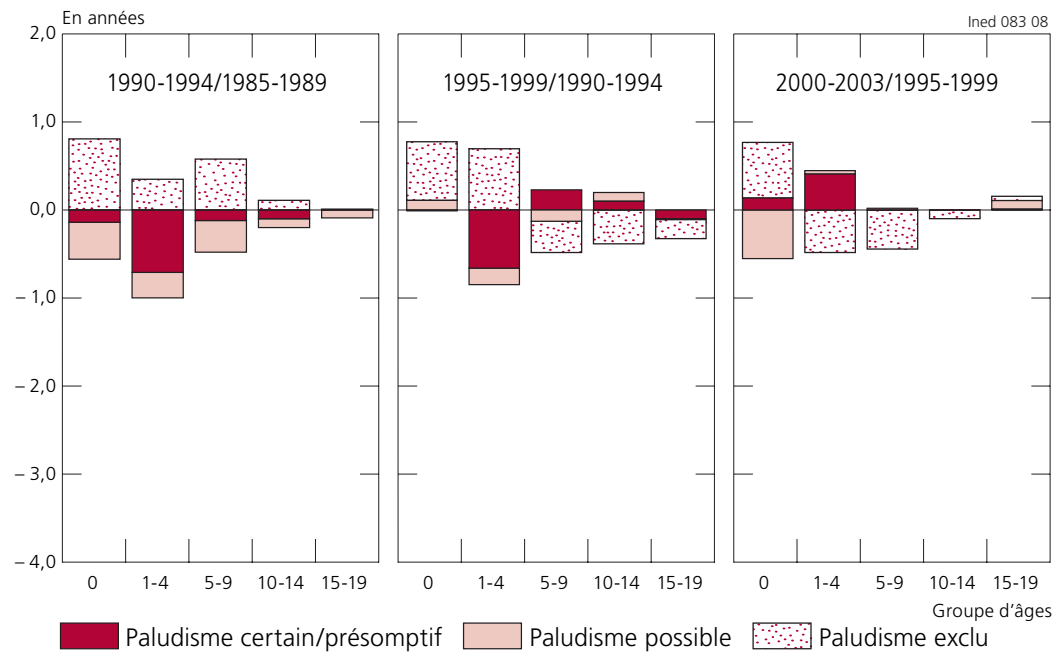

Source : base de données Mlomp, 2005.

Figure 4. Contribution du paludisme par groupe d'âges aux variations de l'espérance de vie féminine à Mlomp

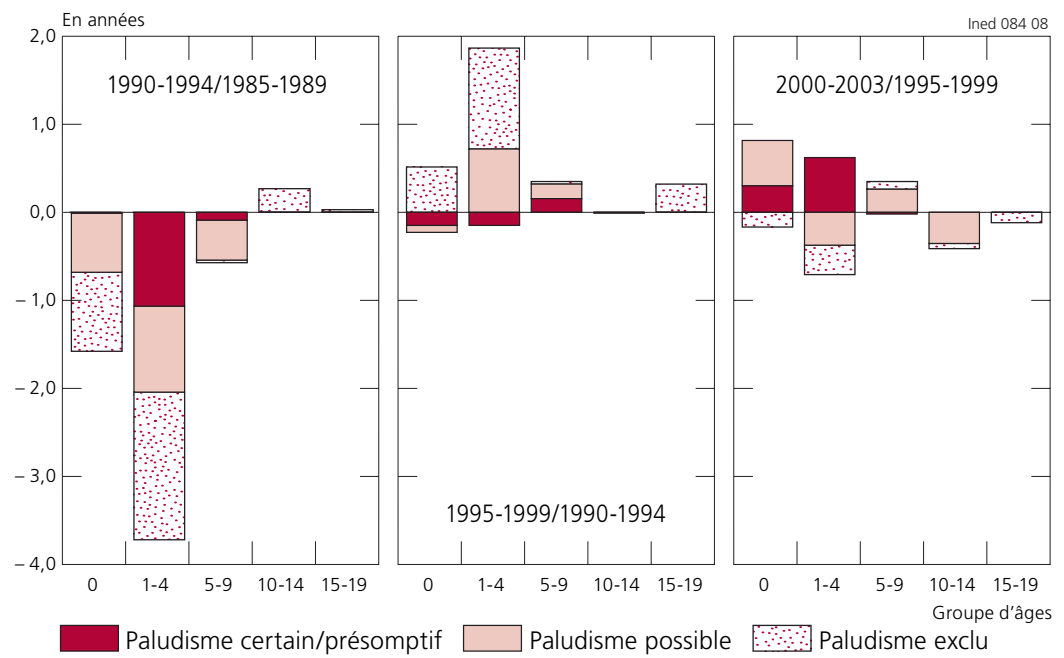

Source : base de données Mlomp, 2005. 


\section{Discussion}

L'évolution de la mortalité palustre à Mlomp est très sensible aux évolutions du contexte épidémiologique et à la réaction des institutions sanitaires. Les médecins en charge de la détermination des causes de décès peuvent être influencés par leur connaissance de ce contexte et ils peuvent donc être poussés ou au contraire réfrénés à diagnostiquer un décès palustre. La lecture recommandée des autopsies verbales par plusieurs médecins avec confrontation des diagnostics a été mise en place à Mlomp en 2004 et la question se pose de son impact sur la détermination des causes de décès. Une analyse réunissant les données de Mlomp et de Bandafassi a montré que la propension à diagnostiquer un décès palustre ne semblait pas avoir changé avec la lecture par deux médecins ; la part des décès de cause mal définie a par contre considérablement diminué, ce qui peut jouer indirectement en cas de redistribution de ces derniers dans les autres catégories (Duthé et al., 2008). Même si cet effet est probablement moindre si on se restreint aux décès de Mlomp du fait des informations médicales, les études à venir sur l'évolution de la mortalité palustre devront tenir compte de ce changement méthodologique.

Le repérage des décès potentiellement palustres nous a permis d'estimer l'impact du paludisme de manière large. À Mlomp, avant l'âge de 20 ans, les décès potentiellement palustres représentent le tiers de la mortalité et cette proportion atteint $44 \%$ si on se restreint à la mortalité juvénile. Des études menées au Kenya, au Nigeria, en Tanzanie et en Gambie ${ }^{(34)}$ ont montré que la réduction voire la disparition du paludisme au sein d'une population entraînait une baisse de la mortalité juvénile de $40 \%$ à $50 \%$, au-delà du seul poids du paludisme dans les causes de décès (Bradley, 1991 ; Payne et al., 1976 ; Molineaux, 1985 ; Alonso et al., 1991). À Mlomp, la hausse de la mortalité palustre certaine ou présomptive s'est bien accompagnée d'une augmentation de la mortalité palustre possible, mais la réduction du paludisme certain ou présomptif constatée au début des années 2000 n'a pas été concomitante d'une baisse de la mortalité possible. La part conséquente des décès de cause mal définie ou inconnue dans notre analyse limite probablement nos analyses.

Enfin, si la recrudescence de la mortalité palustre a bien eu un impact sur la mortalité, elle n'est pas la seule responsable de la hausse de la mortalité au début des années 1990. Durant l'hivernage 1990, une grande cérémonie de circoncision-initiation a été organisée au cours de laquelle les jeunes garçons et les jeunes hommes ont séjourné plusieurs semaines en brousse, ce qui a entraîné un nombre important de décès parmi les garçons à cette période. Mais un pic de mortalité est aussi observé cette année-là pour les filles, probablement lié à une moindre attention des adultes très affairés durant cette période (Duthé, 2006) et, surtout, la mortalité des filles continue d'être plus forte de 1992 à 1994. La recrudescence du paludisme n'explique qu'en partie ce phénomène et une approche en termes de genre est nécessaire pour mieux l'appréhender. 


\section{Conclusion}

L'association du suivi démographique et des expertises médicales à Mlomp contribue incontestablement à la connaissance de l'épidémiologie du paludisme en Afrique et de son impact sur la mortalité : développement de chimiorésistances et efficacité des différents traitements antipaludéens; recrudescence de la mortalité palustre et impact sur la mortalité générale. Mais la particularité de Mlomp tient beaucoup à un suivi sanitaire qui se fait parallèlement au suivi démographique. Des informations médicales existent en milieu rural, au niveau des cases de santé, des dispensaires et des maternités ; mais l'appariement des différentes sources nécessite du temps et est d'autant plus fastidieux que la population suivie est importante. Cette déperdition de l'information médicale, loin d'être exhaustive et complète, reste fort dommageable.

S'agissant de la lutte contre le paludisme, la mise en place des ACT n'est pas immédiate. Plusieurs étapes de tests ont été préalablement nécessaires, puis la multithérapie n’a été appliquée que partiellement, et nécessite maintenant l'adhésion de la population pour être complètement généralisée. Les programmes de santé doivent tenir compte des besoins, des contraintes et des comportements des ménages et des individus qui ont une grande influence sur l'efficacité des stratégies (Coll-Seck, 2003). Les comportements des personnes en matière de recours aux soins, ceux des personnels soignants en matière de prescriptions et d'information et les relations entretenues entre les soignants et les soignés font l'objet d'une attention de plus en plus importante (McCombie, 1996, 2002 ; Williams et Jones, 2004 ; Depoortere et al., 2004 ; Kachur et al., 2004 ; Souares et al., 2006). Dans ce domaine, le suivi démographique et sanitaire constitue un support privilégié pour réaliser des études socio-anthropologiques alliant le qualitatif et le quantitatif.

Remerciements. Je remercie l'Institut national d'études démographiques, le ministère de la Recherche (programme PAL+) et le Muséum national d'histoire naturelle pour leur soutien financier ; Gilles Pison, responsable du programme de recherche du suivi démographique à Mlomp depuis 1985 ; toutes les personnes qui contribuent à la collecte des données et à la mise à jour de la base de données du service des enquêtes de l'Ined et l'équipe de Mlomp, Raphaël Laurent, Emmanuelle Guyavarch, Adama Sow Sambou, Jean-François Trape, Henriette Delenne (Sœur Marie-Joëlle) et Philippe Brasseur. Je remercie également France Meslé, Myriam Khlat et les relecteurs anonymes de la revue pour leurs précieux commentaires. 


\section{RÉFÉRENCES}

Adjuik M., Agnamey P., BABiker A. et al., 2002, « Amodiaquine-artesunate versus amodiaquine for uncomplicated Plasmodium falciparum malaria in African children : a randomised, multicentre trial », The Lancet, 359, p. 1365-1372.

Adjuik M., AgNAmey P., BABiker A. et al., 2004, « Artesunate combinations for treatment of malaria: meta-analysis », The Lancet, 363, p. 9-17.

Adjuik M., SMith T., ClarCK S. et al., 2006, « Cause-specific mortality rates in sub-Saharan Africa and Bangladesh », Bulletin of the WHO, 84(3), p. 181-188.

AgnAmey P., Brasseur P., CiSSÉ M. et al., 2005, « Economic evaluation of a policy change from single-agent treatment for suspected malaria to artesunate-amodiaquine for microscopically confirmed uncomplicated falciparum malaria in the Oussouye District of south-western Senegal », Tropical Medicine and International Health, 10(9), p. 926-933.

AgNAMey P., BRASSEUR P., Eldin DE PeCOUlas P. et al., 2006, « Plasmodium falciparum in vitro susceptibility to antimalarial drugs in Casamance (south-western Senegal) during the first 5 years of routine use of artesunate-amodiaquine », Antimicrobial Agents and Chemotherapy, 50(4), p. 1531-1534.

AlONSO P., LindSAY S., ARMSTRONG J. et al., 1991, « The effect of insecticide-treated bednets on mortality of Gambian children », The Lancet, 337, p. 1499-1502.

AMAT-Roze J.-M., 2002, « Aspects de la géographie du paludisme », Information géographique, 65(3), p. 236-243.

ANDREEV E.M., SHKOlNikOV V.M., BEGUN A.Z., 2002, « Algorithm for decomposition of differences between aggregate demographic measures and its application to life expectancies, healthy life expectancies, parity-progression ratios and total fertility rates », Demographic Research, 7(14), p. 500-521.

ANKER M., 1997, « The effect of misclassification error on reported cause-specific mortality fractions from verbal autopsy », International Journal of Epidemiology, 26(5), p. 1090-1096.

Anker M., Black R.E., Coldham C. et al., 1999, A Standard Verbal Autopsy Method for Investigating Causes of Death in Infants and Children, Genève, OMS.

BARBIERI M., 1989, «The determinants of infant and child mortality in Senegal : An analysis of DHS data », Thèse de doctorat de démographie, Berkeley, Université de Californie.

BAUdON D., 2000, « Les paludismes en Afrique sub-saharienne », in Gruénais M.-E. et Pourtier R. (dir.), «La santé en Afrique. Anciens et nouveaux défis », Afrique contemporaine, 195, juil-sept, p. 36-45.

BAYA B., 2004, « Population et maladies infectieuses au Burkina Faso », communication présentée au séminaire HIV, Resurgent Infections and Population Change in Africa, IUSSP/UIESP, 12-14 février, Burkina Faso, Ouagadougou.

Berkley J.A., Mwangi I., Mellingtion F. et al., 1999, «Cerebral malaria versus bacterial meningitis in children with impaired consciousness », Quarterly Journal of Medicine, 92, p. 151-157.

BIRAUD Y., 1956, Méthode pour l'enregistrement par des non-médecins des causes élémentaires de décès dans des zones sous-développées, Genève, OMS. 
BRADlEy D.J., 1991, « Morbidity and mortality at Pare-Taveta, Kenya and Tanzania, 1954-66 : The effects of a period of malaria control », in Feachem R.G., Jamison D.T. (éd.), Disease and Mortality in sub-Saharan Africa, The World Bank, Oxford, Oxford University Press, p. 248-263.

Brasseur P., GuiguemdÉ R., DiAllo S. et al., 1999, « L'amodiaquine reste efficace dans le traitement du paludisme non compliqué en Afrique occidentale et centrale », Transactions of the Royal Society of Tropical Medicine and Hygiene, 93(6), p. 645-650.

Chandramohan D., Maude G.H., Rodrigues L.C., Hayes R.J., 1994, « Verbal autopsies for adult deaths: Issues in their development and validation », International Journal of Epidemiology, 23, p. 213-222.

Chandramohan D., Setel P., Quigley M., 2001, « Effect of misclassification of causes of death in verbal autopsy : Can it be adjusted ? ", International Journal of Epidemiology, 30, p. 509-514.

Chandramohan D., Soleman N., Shibuya K., Porter J., 2005, « Ethical issues in the application of verbal autopsies in mortality surveillance systems », Tropical Medicine and International Health, 10(11), p. 1087-1089.

Cissé M., Duthé G., SOKHna C et al., 2005, « First line treatment of malaria whith artemisinin-class combination therapy (ACT) dramatically reduces malaria mortality in Senegal ", Poster présenté au colloque international Medicine and Health in the Tropics, 11-14 septembre, Marseille.

COLl-SECK A.M., 2003, in « Special Report», The Lancet, 361(9353), p. 234.

Delaunay V., 2002, « Apports et limites de l'observation continue. Le suivi de population de Niakhar au Sénégal », in Baya B., Willems M. (éd.), L’apport des approches renouvelées pour l'analyse du début de la transition démographique, Paris, Ceped (Les documents et manuels du Ceped $\left.n^{\circ} 13\right), 138$ p.

Depoortere E., Guthmann J.-P., Sipilanyambe N. et al., 2004, « Adherence to the combination of sulphadoxine-pyrimethamine and artesunate in the Maheba refugee settlement, Zambia ", Tropical Medicine and International Health, 9(1), p. 62-67.

Desgrées Du Loû A., Pison G., SAmb B., Trape J.-F., 1996, « L'évolution des causes de décès d'enfants en Afrique : une étude de cas au Sénégal avec la méthode d'autopsie verbale », Population, 4-5, p. 845-882.

DUTHÉ G., 2006, « La transition sanitaire en milieu rural sénégalais. Évolution de la mortalité à Mlomp depuis 1985 et influence du paludisme chimiorésistant », Thèse de doctorat de démographie, Paris, Muséum national d'histoire naturelle.

Duthé G., FAye S., GuYAVARCh E., et al., 2008, « La détermination des causes de décès par autopsie verbale : étude de la mortalité palustre en zone rurale sénégalaise », Paris, Ined (Documents de travail n ${ }^{\circ} 150$ ), 35 p. + annexes.

ENEl C., Pison G., Lefebvre M., 1993, « De l'accouchement traditionnel à l'accouchement moderne au Sénégal », Cahiers Santé, 3, p. 441-446.

FAUVEAU V., 2002, "L'observation des causes de décès dans les pays en développement », in Caselli G., Vallin J., Wunsch G. (dir.), Démographie : analyse et synthèse. Volume III - Les déterminants de la mortalité, Paris, Ined, p. 81-99.

Garenne M., Cantrelle P., 1997, « Three decades of research on population and health : The ORSTOM experience in rural Senegal, 1962-1991 ", in Das Gupta M., Aaby P., Garenne M., Pison G. (éd.), Prospective Community Studies in Developing Countries, Oxford, Clarendon Press/Oxford University Press, p. 233-252.

GARENne M., FAUVEAU V., 2006, « Potential and limits of verbal autopsies », Bulletin of the WHO, 84(3), p. 164-165. 
GARENnE M., FOnTAine O., 1988, « Enquête sur les causes probables de décès en milieu rural sénégalais », in Vallin J., D'Souza S., Palloni A. (éd.), Mesure et analyse de la mortalité. Nouvelles approches, Paris, Ined (Travaux et Documents, Cahier $\mathrm{n}^{\circ}$ 119), p. 123-141.

GREGSON A., Plowe C.V., 2005, « Mechanisms of resistance of malaria parasites to antifolates », Pharmacological Reviews, 57(1), p. 117-145.

HilL K.H., 1999, « The measurement of adult mortality : an assessment of data availability, data quality and estimation methods ", in Chamie J., Cliquet R. (éd.) Health and Mortality Issues of Global Concern. Proceedings of the symposium on Health and Mortality, Brussels, 19-22 Novembre 1997, Bruxelles, Population and Family Study Centre, Flemish Scientific Institute / New York, Division de la Population, Département des affaires économiques et sociales des Nations unies, p. 72-83.

INDEPTH NETWORK, à paraître, Causes of Death at INDEPTH Sites.

KACHUR S.P., KHATIB R.A., KAIZER E. et al., 2004, « Adherence to antimalarial combination therapy with sulfadoxine-pyrimethanine and artesunate in rural Tanzania ", American Journal of Tropical Medicine and Hygiene, 71(6), p. 715-722.

KAhn K., Tollman S.M., GAREnne M., GeAR J.S., 2000, « Validation and application of verbal autopsies in a rural area of South Africa », Tropical Medicine E International Health, 5(11), p. 824-831.

LANGFORD C., 1996, « Reasons for the decline in mortality in Sri Lanka immediately after the Second World War : A re-examination of the evidence », Health Transition Review, 1, p. 3-24.

LOHLÉ-TART L., FRANÇOIS M., 1999, État civil et recensements en Afrique francophone. Pour une collecte administrative de données démographiques, Paris, Ceped (Les documents et manuels du Ceped n $\left.{ }^{\circ} 10\right), 564$ p.

MARA/ARMA, 1999, Vers un atlas du risque de la malaria en Afrique. Premier rapport technique de la collaboration MARA/ARMA, Durban, 1998 (http://www.mara.org. $\mathrm{za} /$ ).

MARQUET I., 2003, « Accessibilité aux antipaludiques au Sénégal. Effets de l'introduction de l'association artesunate/amodiaquine », mémoire de DESS de santé publique, Paris, École nationale de santé publique.

Marsh K., English M., Crawley J., Peshu N., 1996, « The pathogenesis of severe malaria in African children », Annals of Tropical Medicine and Parasitology, 90(4), p. 395-402.

Mathers C.D., Lopez A.D., Murray C.J.L., 2006, « The burden of disease and mortality by condition : Data, methods, and results for 2001 », in Lopez A.D., Mathers C.D., Ezzati M. et al. (éd.), Global Burden of Disease and Risk Factors, Washington, The World Bank / New York, Oxford University Press, p. 45-240.

Maude Gillian H., Ross David A., 1997, « The effect of different sensitivity, specificity, and cause-specific mortality fractions on the estimation of differences in cause-specific mortality rates in children from studies using verbal autopsies », International Journal of Epidemiology, 26(5), p. 1097-1106.

MCCOMBIE S.C., 1996, « Treatment seeking for malaria : A review of recent research », Social Science and Medicine, 43(6), p. 933-945.

MCCOMBIE S.C., 2002, «Self-treatment for malaria: The evidence and methodological issues », Health Policy and Planning, 17(4), p. 333-344.

MESLÉ F., 2002, « Les causes médicales de décès », in Caselli G., Vallin J., Wunsch G. (dir.), Démographie : analyse et synthèse. Volume III - Les déterminants de la mortalité, Paris, Ined, p. 53-79. 
MOlineauX L., 1985, "La lutte contre les maladies parasitaires : le problème du paludisme, notamment en Afrique », in Vallin J., Lopez A. (éd.), La lutte contre la mort, Paris, Puf (Travaux et Documents, Cahier n ${ }^{\circ}$ 108), p. 11-40.

NATIONS UNIES, 2002, Methods for estimating adult mortality, New York, Division de la Population, Département des affaires économiques et sociales des Nations unies.

NATIONS UNIES, 2005, World Population Prospects. The 2004 Revision, New York, Division de la Population, Département des affaires économiques et sociales des Nations unies.

NATIONS UniES, 2007, World Population Prospects. The 2006 Revision, New York, Division de la Population, Département des affaires économiques et sociales des Nations unies.

Ndiaye S., AyAd M., 2006, Enquête démographique et de santé Sénégal 2005, Calverton, Maryland, CRDH (Sénégal) et ORC Macro.

OMS, 1977, Classification internationale des maladies, $9^{e}$ révision (1975), Genève, OMS.

OMS, 1998, Roll Back Malaria Project : Resources Support Network for Prevention and Control of Malaria Epidemics, Genève, OMS.

OMS, 2005, Rapport mondial 2005 sur le paludisme, Genève, OMS.

PAYNE D., 1987, «Spread of chloroquine resistance in Plasmodium falciparum », Parasitology Today, 3, p. 241-246.

PAyne D., Grab B., Fontain R.E., Hempel J.H.G., 1976, « Impact of control measures on malaria transmission and general mortality », Bulletin of the WHO, 54, p. 369-377.

PISON G., 2005, « Population observatories as sources of information on mortality in developing countries », Demographic Research, 13, p. 301-334.

Pison G., Desgrées du Lồ A., LANGAney A., 1997, « Bandafassi : a 25 years prospective community study in rural Senegal (1970-1995) », in Das Gupta M., Aaby P., Garenne M., Pison G. (éd.), Prospective Community Studies in Developing Countries, Oxford, Clarendon Press/Oxford University Press, p. 253-275.

PisOn G., Duthé G., GuYAVARCh E. et al., 2005, « La mortalité violente au Sénégal : niveaux et causes dans trois zones rurales ", communication présentée au Congrès international de la population, UIESP/IUSSP, 18-23 juillet, Tours, France.

PISON G., ENEL C., 2005, « Le passage à l'âge adulte et la constitution de la famille. Évolutions récentes à Mlomp (Sénégal) », in Vignikin K., Vimard P. (dir.), Familles au Nord. Familles au Sud, Louvain-la-Neuve, Academia Bruylant, p. 155-177.

PisOn G., ENEL C., GABAdinho A. et al., 2001, « Migrations saisonnières, sexualité et fécondité. Une étude de cas dans la zone rurale de Mlomp, au Sénégal », communication présentée au colloque international Genre, population et développement en Afrique, ENSEA/Iford/Ined/UEPA, 16-21 juillet, Abidjan, Côte d'Ivoire.

Pison G., Kodio B., GuYAVArch E., ÉTARD J.-F., 2000, « La mortalité maternelle en milieu rural au Sénégal », Population, 6, p. 1003-1018.

Pison G., Trape J.-F., LefebVRe M., Enel C., 1993, « Rapid decline in child mortality in a rural area of Senegal », International Journal of Epidemiology, 22(1), p. $72-80$.

Pison G., Wade A., Gabadinho A., Enel C., 2002, « Mlomp DSS, Senegal », in Indepth network, Population and Health in Developing Countries (Volume 1), Ottawa, International Development Research Centre, p. 271-278.

Rogier C., Fusaï T., Pradines B., Trape J.-F., 2005, « Comment évaluer la morbidité attribuable au paludisme en zone d'endémie ? ", Revue d'épidémiologie et de santé publique, 53, p. 299-309. 
ROWE A.K., 2005, « Should verbal autopsy results for malaria be adjusted to improve validity? », International Journal of Epidemiology, 34(3), p. 712-713.

ROWE A.K., ROWE S.Y., SNOW R.W. et al., 2006, « The burden of malaria mortality among African children in the year 2000 », International Journal of Epidemiology, 35(3), p. 691-704.

SETEl P.W., Whiting D.R., Hemed Y. et al., 2006, « Validity of verbal autopsy procedures for determining cause of death in Tanzania », Tropical Medicine and International Health, 11(5), p. 681-696.

SIBLEY C.H. et al., 2001, «Pyrimethamine-sulfadoxine resistance in Plasmodium falciparum: What next? », Trends in Parasitology, 17, p. 582-588.

Smith T., KilleEn G., Lengeler C., TAnner M., 2004, « Relationship between the outcome of Plasmodium falciparum infection and the intensity of transmission in Africa », American Journal of Tropical Medicine and Hygiene, 71(2s), p. 80-86.

SMith T., Ross A., MAIRE N. et al., 2006, « An epidemiologic model of severe morbidity and mortality caused by Plasmodium falciparum », American Journal of Tropical Medicine and Hygiene, 75(2 suppl.), p. 63-73.

SNOW R.W., Armstrong J.R., FORSTER D. et al., 1992, « Childhood deaths in Africa : Uses and limitations of verbal autopsies », The Lancet, 340(8815), p. 351-355.

SnOw R.W., Craig M., Deichmann U., LE Sueur D., 1999a, « A preliminary continental risk map for malaria mortality among African children », Parasitology Today, 15, p. 99-104.

SNOW R.W., Craig M., Deichmann U., MARSH K., 1999b, « Estimating mortality, morbidity, and disability due to malaria among Africa's nonpregnant population ", Bulletin of the WHO, 77, p. 624-640.

Sokhna C., Molez J.-F., NdiAye P. et al., 1997, « Tests in vivo de chimiosensibilité de Plasmodium falciparum à la chloroquine au Sénégal : évolution de la résistance et estimation de l'efficacité thérapeutique », Bulletin de la Société de pathologie exotique, 90(2), p. 83-89.

Soleman N., Chandramohan D., Shibuya K., 2006, « Verbal autopsy: Current practices and challenges », Bulletin of the WHO, 84(3), p. 239-245.

SouAres A., LAlOu R., SENE I. et al., 2006, « Connaissances et pratiques des agents de santé de la région de Thiès concernant la nouvelle thérapie des accès palustres », Santé publique, 18(2), p. 93-104.

Todd J.-E., De Francisco A., O'Dempsey T.J., Greenwood B.M., 1994, « The limitations of verbal autopsy in a malaria-endemic region », Annals of Tropical Paediatrics, 14(1), p. 31-36.

TRAPE J.-F., 2001, « The public health impact of chloroquine resistance in Africa ", American Journal of Tropical Medicine, 64(1-2)S, p. 12-17.

TRAPE J.-F., PISON G., PREZIOSI M.-P. et al., 1998, « Impact of chloroquine resistance on malaria mortality », Comptes rendus de l'Académie des sciences, Série Sciences de la vie, 321, p. 689-697.

TrApe J.-F., PisOn G., SPIEGEl A. et al., 2002, « Combating malaria in Africa », Trends in Parasitology, 18(5), p. 224-230.

UNICEF, 2002, « L'enregistrement à la naissance : un droit pour commencer », Digest Innocenti, 9, $34 \mathrm{p}$.

WiLliAMS H.A., JONES C.O.H., 2004, « A critical review of behavioral issues related to malaria control in sub-Saharan Africa: What contributions have social scientists made?", Social Science and Medicine,59(3), p. 501-523. 


\section{GÉRALDine Duthé • ReCRUdescence du PALUdisme au SénéGAL : LA MESURE de LA MORTALITÉ PALUSTRE À MLOMP}

Le paludisme est l'une des principales causes de mortalité des enfants en Afrique au sud du Sahara. Or, la lutte contre cette maladie est complexe - avec le développement de résistances des parasites aux traitements administrés - et le manque de données démographiques et sanitaires dans les pays les plus touchés empêche son évaluation. Les sites de suivi démographique permettent de mesurer les niveaux et tendances de la mortalité et des causes de décès. Les données qu'ils fournissent présentent toutefois des limites, surtout dans le cas du paludisme. Au Sénégal, le site de Mlomp permet de coupler les informations collectées auprès des habitants avec celles des institutions sanitaires locales, et donc d'étudier précisément la mortalité palustre. Celle-ci, très faible à la fin des années 1980, a augmenté suite au développement d'une résistance du Plasmodium falciparum à la chloroquine, le traitement couramment utilisé et jusque-là efficace. L'introduction de nouveaux traitements au début des années 2000 a bien permis de réduire la mortalité palustre diagnostiquée mais l'adoption d'une définition large des décès attribuables au paludisme montre qu'elle représenterait encore une part importante de la mortalité.

\section{Géraldine Duthé • Malaria Resurgence in Senegal: Measuring Malaria MORTALITY IN MLOMP}

Malaria is one of the leading causes of child mortality in sub-Saharan Africa. With the development of drugresistant parasites, the fight against malaria has become complex, and because demographic and health data are scarce in the most hard-hit countries, the impact of the disease is difficult to evaluate. Demographic surveillance sites provide a means to measure levels and trends in mortality and causes of death. The data they provide are not exhaustive, however, for malaria in particular. At the Mlomp site in Senegal, information from inhabitants can be matched against data from local health facilities for more precise study of malaria mortality. From very low levels in the late 1980s, malaria mortality increased as the Plasmodium falciparum became resistant to chloroquine, the standard drug which, until then, had been an effective treatment. Although the introduction of new treatments in the early 2000s reduced diagnosed malaria mortality, the adoption of a broad definition of deaths attributable to malaria shows that the disease still accounts for a large share of mortality.

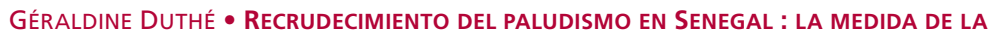 MORTALIDAD PALÚDICA EN MLOMP}

El paludismo es una de las principales causas de mortalidad de los niños en África al sur del Sahara. Ahora bien, la lucha contra esta enfermedad es compleja - con el desarrollo de resistencias de los parásitos a los tratamientos administrados - y la falta de datos demográficos y sanitarios en los países más afectados impide su evaluación. Los centros de seguimiento demográfico permiten medir los niveles y tendencias de la mortalidad y de las causas de muertes. Los datos que proporcionan presentan sin embargo límites, sobre todo en el caso del paludismo. En Senegal, el centro de Mlomp permite aparejar las informaciones recogidas ante los habitantes con las de las instituciones sanitarias locales y por lo tanto estudiar con precisión la mortalidad palúdica. Esta, muy reducida al final de los años 1980, ha aumentado a raíz del desarrollo de una resistencia del Plasmodium falciparum a la cloroquina, el tratamiento corrientemente utilizado y hasta la fecha eficaz. La introducción de nuevos tratamientos a principios de los años 2000 ha permitido efectivamente reducir la mortalidad palúdica diagnosticada pero la adopción de una definición amplia de las muertes debido al paludismo muestra que representaría todavía una proporción importante de la mortalidad.

Géraldine DutHÉ, Institut national d'études démographiques, 133 boulevard Davout, 75980 Paris Cedex 20, France, tél : 33 (0)1 560622 47, courriel : geraldine.duthe@ined.fr 